\title{
Functional Neurological symptoms: Optimising efficacy of inpatient treatment and preparation for Change using the Queen Square Guided Self-Help (QGSH)
}

\author{
*Susan Humblestone ${ }^{1}$ \& *Jacob Roelofs ${ }^{2}$, Caroline Selai1, ${ }^{1,2}$ \& Michael Moutoussis ${ }^{1,3,4}$ \\ 1 - Neuropsychiatry Dept, National Hospital for Neurology and Neurosurgery, Queen Square, \\ London, UK
}

2 - Dept of Clinical and Movement Neuroscience, UCL Queen Square Institute of Neurology, London, WC1N 3BG, United Kingdom

3 - Wellcome Centre for Human Neuroimaging, University College London, London WC1N 3BG,

United Kingdom

4- Max Planck - University College London Centre for Computational Psychiatry and Ageing

Research, London WC1B 5EH, United Kingdom

*Joint first authors

Word Count: 5503, 3 tables and 3 figures, 39 citations. (6690 including references)

\section{$\underline{\text { Precis }}$}

We describe the development and preliminary evaluation of a brief, remotely delivered psychotherapeutic intervention for patients with Functional Neurological Symptom Disorder (FNSD) and further development of remotely delivered interventions for this patient group.

\section{Abstract}

Objective: Functional Neurological Symptoms (FNS) are disabling symptoms without macrostructural cause. While inpatient treatment confers important benefits, it is resource intensive and hence it is important to optimize its efficiency.

Methods: We developed a brief, internet-based preparatory therapy based on psychoeducation and $\mathrm{CBT}$, termed the Queen Square Guided Self-help (QGSH) to maximize the efficacy of the inpatient FNS treatment at the National Hospital for Neurology and Neurosurgery.

Results: The QGSH aims to ensure that prior to admission the patient understands 1) The diagnosis of FNS , 2) The five areas CBT model and 3) The use of Goal setting in rehabilitation. It has now run since 2017 and 191 patients have taken part in the inpatient FNS program, with 122 of these have participated in the QGSH. It runs for up to twelve weeks and includes original videos and patient worksheets, as well as signposting to existing published resources. Information is sent weekly by email and content is delivered in the form of eleven modules built around online video sessions.

Conclusion: We believe that the set of materials used in QGSH has the potential to benefit patients with FNS and can support clinicians wishing to develop their expertise. It could help with the 
development of new FNS services, and we are in the process of developing it into a standalone service. We hope that the experience of the Queen Square team can be used to help patients and clinicians to improve the provision of FNS services.

Keywords: Functional Neurological Symptoms, Guided Self-Help, Cognitive Behavioural Therapy, Rehabilitation.

Abbreviations: FNS: Functional Neurological Symptoms GSH: Guided self-help, CBT: Cognitive Behavioural Therapy, QGSH: Queen Square Guided Self-help, NHNN: National Hospital for Neurology and Neurosurgery

Conflicts of Interest and Source of Funding: None of the authors have a Conflict of Interest to declare. The Wellcome Trust Centre for Human Neuroimaging is funded by the Wellcome Trust. The Max Planck - University College London Centre for Computational Psychiatry and Ageing Research is is a joint initiative of the Max Planck Society and UCL. M. Moutoussis receives support from the NIHR UCLH Biomedical Research Centre.

\section{Background}

Functional Neurological Symptoms (FNS) are disabling symptoms without macro-structural cause (1). Previously these symptoms have been described using a range of terms including 'hysterical', 'conversion' and 'somatisation', each with implications about the underlying mechanism. The variety of terms has left patients 'muddled', and can be found offensive (2). 'Functional' is now preferred as it is acceptable to patients and does not assume an underlying mechanism (3).

The diagnosis is based on a pattern of positive signs and symptoms characteristic of functional disease, and which vary with time or attention (4). This is reflected in the DSM V and ICD-10 criteria (see Appendix 1). FNS may include motor symptoms, sensory disturbance, or other neurological symptoms such as aphonia. An important subtype of FNS is non-epileptic attack disorder (NEADs), also known as dissociative or psychogenic seizures, which present with episodes of disrupted consciousness without EEG evidence of epileptic brain activity (1).

FNS causes a similar level of disability to 'organic' neurological disorders but with a higher rate of psychiatric comorbidity (5). It is thought to account for between $6-16 \%$ of all neurology clinic referrals (6) with an incidence of 4-12 per 100,000 (7). 'Functional overlay', where functional symptoms co-exist with 'organic' illnesses, are thought to occur in up to $30 \%$ of neurology patients (6) and it is thought that $10-50 \%$ of patients with epilepsy may have a combination of epileptic and non-epileptic seizures (8). We place 'organic' in quotes to emphasize that all disorders of brain function have an organic substrate at the microscopic level of synaptic connectivity and 
neurotransmitter function. Similarly, we avoid referring to FNS as having 'no structural cause', preferring to talk about 'no macro-structural cause' as might be detected by current medical imaging.

The term 'organic' is commonly used to refer to disorders with well-established histopathological or neuro-chemical substrate but its simplistic application in the case of FNS has caused many misunderstandings, which the program described in this article often have to address. Importantly, psychological factors and negative life events are thought to be part of a process of symptom emergence, contributing to disrupted attentional and emotional processing to produce and maintain symptoms (9). Yet the role of psychological factors is controversial, as many perceive clinical formulations at the psychological or psychiatric level as discounting true distress, disbelieving or stigmatizing sufferers as 'mad' and denying the role of the brain - while of course they simply refer to a different level of brain function and structure, that of information processing and learning.

Regarding the place of information and learning in treatment, there is significant evidence that the quality of diagnostic explanation impacts the efficacy of treatment (10) and a substantial minority of patients are symptom-free after the initial consultation alone (11). Additionally, patients feeling they are believed by their doctor is an important factor in their recovery (12). Patient acceptance of the diagnosis, acknowledgement that emotion may play an important role in symptom production and a stable social environment all increase the chance of a good recovery $(13,14)$. On the other hand, poor outcomes are associated with expectation of non-recovery, non-attribution of symptoms to psychological factors and receipt of health-related benefits (15). Psychiatric co-morbidity, particularly personality disorder are also important negative predictive factors (16). This may reflect the difficulty of treatment in the presence of another disorder, and in personality disorders, difficulties with collaboration with treatment.

\section{The multidisciplinary approach}

Evidence supports both psychological and physical interventions (17-19), helping to overcome the difficulties from diagnosis to effective treatment that have historically troubled the management of FNS (20). Acceptance of this multidisciplinary approach is illustrated by $55 \%$ of neurologists and $88 \%$ of psychiatrists favouring a combined treatment in one study (21). Using a multidisciplinary team (MDT) of health professionals from a range of backgrounds can maximise the impact of each form of therapy by working co-operatively (22). This approach is supported by both retrospective (23) and prospective case series (19). MDT treatment has also been shown to be effective at treating functional gait disorders and NEADs, as well as functional motor symptoms (24).The National Hospital for Neurology and Neurosurgery (NHNN) offers a tertiary care, multidisciplinary treatment 
package for FNS, whose centrepiece is the inpatient programme. Referred patients must have a clear diagnosis of FNS, accept their diagnosis, and agree to therapeutic goals of improving daily function and increasing activity. Rehabilitation is goal-oriented and implemented by a team of specialist nurses, occupational therapists, physiotherapists, cognitive behaviour therapists and neuropsychiatrists. The program is organised around weekly goal setting, ward rounds, a combination of single and integrated therapy sessions and a family meeting held in the final week. The length of inpatient admissions used to be based on clinical judgement, depending on progress towards goals. The average stay was thus approximately 12 weeks, and a majority of patients achieved good outcomes. However, service pressures meant that the length of stay had to be limited to four weeks.

\section{The need for Guided Self-Help}

While inpatient MDT treatment confers important benefits (19) it is resource intensive and hence under pressure to minimize its length. The shortening of admissions in NHNN meant that many patients spent a significant proportion of their admission gaining an understanding of the diagnosis and the rehabilitative approach. Many expected an admission based on 'organic' investigations and medical intervention, and had doubts about the biopsychosocial, goal-driven approach and how important self-management would be for effective rehabilitation. By the time a collaborative understanding of FNS has been achieved, there was often little time left for hands-on rehabilitation. Thus we aimed to develop a preparatory therapy, termed the 'Queen Square Guided Self-help' (GGSH) based on psychoeducation and CBT and to institute it as a key part of the treatment package, so that patients would to make the best use of the inpatient treatment.

\section{Methods}

We developed the QGSH through a) considering the multidisciplinary approach that patients needed to learn about, b) adapting existing guided self-help approaches, c) incorporating an on-going process of service evaluation and finally, d) aiming to provide the resources we developed to the community.

\section{Key therapies within the MDT}

Here, we briefly describe the contribution of physical, psychological, occupational, psychiatric, and whole-team contributions to our program.

Specialist physiotherapy for motor FNS often focuses on retraining abnormal movements(25), and specifically on reducing attention on the affected limb. This reducing of attention is not typical of traditional physiotherapy. Specialist physiotherapy can be highly effective, especially for motor FNS, 
with $82 \%$ of patients in one study responding (26). Significant improvements in physical function and quality of life sustained over follow-up have been seen $(24,27)$. Specialised PT programs focus on patient education, exploring symptoms and producing coping strategies while developing a patient's capacity for self-management.

Psychologically, we use Cognitive behavioural therapy (CBT) for FNS (28) within a broad biopsychosocial approach. The cognitive component aims to modify the patient's unhelpful beliefs in relation to their illness (29). CBT has been found to be effective in studies looking at both in one-toone settings (17) and groups (18). In QGSH, using experienced clinicians supported by a multidisciplinary approach means that each patient's needs are understood in the light of a broad range of knowledge, including psychodynamic and systemic hypotheses. A range of psychological therapies have been used for FNS, including Brief Psychodynamic interpersonal therapy (BPIP), which is also supported by some evidence (30).

Occupational therapy for FNS is primarily concerned with function rather than impairment. This means that a person's engagement in daily activities, their occupation in the broadest sense, is the focus of intervention rather than symptom resolution per se, although activity itself can also lead to significant improvements. Therapy is concerned with understanding the meaning of activity to an individual under the domains of self-care, work, and leisure. Problem solving to resolve barriers the individual experiences in participating in these activities is therefore central. An in-depth history taken from the individual allows the therapist to place them in the context of their wider biopsychosocial environment. Understanding a person's narrative is essential in planning treatment. As Allied health professionals trained in both physical and mental health, but focused on rehabilitation and recovery, occupational therapists are well suited for treating people with FNS (31). In an inpatient setting the 24 -hour care provided by registered nurses is important in providing a consistent environment in which the patient is able to work on their goals. This is particularly important as in-patient rehabilitation work often involves therapeutic risk taking. The nursing team also provide the detailed knowledge of a person's presentation throughout the day which allows for a 24-hour dynamic risk assessment and so supports a therapeutic rehabilitative environment.

Occupational therapy, along with nursing, has a key role in addressing the stigma and trauma which patients with FNS often experience. Professionals' focus on 'negative' diagnostic tests can leave them feeling that they are not believed by doctors or by other people in their lives. A chain of onward referrals eventually leading to a psychologist or psychiatrist can produce the impression that their symptoms are being dismissed as 'all in their head', further straining their relationship with healthcare providers. Addressing this and rebuilding a positive therapeutic relationship is central to 
the therapy for FNS and it is important that a basic biopsychosocial explanation of the illness is given early, rather than the process conveying psychosocial factors as avoided explanations of last resort.

Expert psychiatric understanding is also important. Many of the more disabled FNSD patients present with a range of functional symptoms in the presence of comorbid 'organic' diseases. A significant proportion present with psychiatric co-morbidity, not only anxiety and depression but other psychiatric disorders, and may have a complex biopsychosocial presentation whose mental and somatic health components are difficult for non-specialists to delineate.

\section{The development of Guided Self-Help}

Self-help approaches have been established for many decades in the psychological therapies (32). These include, for example, Bibliotherapy, where patients read recommended books and forms of Computerised CBT delivered by CD or DVD or via the internet. 'Book prescription' schemes where patients can borrow on extended loan specific books via a 'prescription' from a health professional is one way of making bibliotherapy more accessible. In advocating greater accessibility and flexibility in modes of therapy delivery, Lovell \& Richard (2000) advocated for Multiple Access Points and Levels of Entry to therapy (MAPLE) (33). Thus, as well as part of an integrated care package, Guided self-help may form one stage in a stepped-care programme. Indeed, NHS Scotland has advocated 'Stepped Care' for patients with FNS. Step 1 is Diagnosis; Step 2 is a brief intervention; Step 3: complex care with a Multi-Disciplinary Team (34).

Cuijpers and Schuurmans reviewed the history of self-help interventions for anxiety disorders and outlined the forms it can take (See Table 1). The only RCT trial to investigate the efficacy of guided self-help for FNS was performed by Sharpe et al , providing class III evidence. Participants allocated to the UC + GSH condition showed greater improvement in the CGI with an odds ratio of 2.36 (95\% $\mathrm{Cl}: 1.17-4.74, \mathrm{p}=0.016)$. There was a $13 \%$ absolute improvement in proportion rating their health as 'better' or 'much better', translating to a number needed to treat of 8 . Much of the recent work on GSH has moved to internet-based approaches, which can amplify the power of GSH.

Unguided self-help: Provided by a book or electronically via the internet or computer programs. There is no professional support of either the user's understanding of the method or as to how far to pursue it.

Self-help as part of face-to-face therapy: Here it can be used as part of regular treatment with a professional providing the patient with a self-help materials to speed up the treatment process or to give them an opportunity to practice components of the therapy independently. For example, self-help sleep-hygiene guides are commonly used in standard CBT.

Self-help as an independent intervention: The patient works through a self-help workbook or worksheet with support from a professional at regular times. These are usually brief contacts aimed to provide added explanation about the methods where needed rather than developing a traditional patient-therapist relationship. The capacity for this has expanded significantly with the development of the internet. 
In this light, the_QGSH was developed as a brief therapeutic intervention, which aims to ensure that prior to admission the patient understands 1) The diagnosis of FNS and how their own diagnosis has been reached, 2) The five areas CBT model and has started practicing it, 3) The use of Goal setting in rehabilitation. The 'Five-areas' approach (36) focuses on psychoeducation, explains FNS within a biopsychosocial model and teaches goal-oriented self-management to support engagement with the inpatient program. The most important aim, however, is to develop a collaborative, trusting alliance with the neuropsychiatry multidisciplinary team.

\section{Service Evaluation}

In order to improve the QGSH, we developed an ongoing evaluation system based on a Patientprovided Routine Outcome Measure (PROM) and a complementary Clinician-provided Routine Outcome Measure (CROM). These were both produced in four stages: assembling items, preliminary scale evaluation, analysis of reliability and validity and final clinical evaluation, in line with standard development of psychometric scales.

\section{Results}

The Referral process - Patients are referred to the overall FNS service by neurologists and neuropsychiatrists who have established the diagnosis and are first seen in a neuropsychiatry MDT clinic to assess their suitability for treatment. This clinic consists of a Neuropsychiatrist, FNS specialist nurse, FNS specialist occupational therapist and a FNS specialist physiotherapist. When they are first seen in this clinic some patients have accepted their diagnosis while other patients report they do not recall their diagnosis or reason for referral. The decision to admit is then made collaboratively based on each patient's needs and the MDT assessment.

The referral process is outlined in Figure 1, and the criteria to be considered for treatment are:

1) Definitive diagnosis of FNS by a Neurologist

2) Acceptance of the diagnosis, with no requests for further diagnostic investigations.

3) Willingness to engage in MDT programme

4) Ability to work with a goal orientated approach

5) No current litigation related to symptoms (though this is on a case by case basis)

The MDT clinic assessment has a number of roles in additional to the usual clinical history taking. The lead clinician must build trust, take an inventory of symptoms, explain the diagnosis and the approach to treatment. During the assessment interviewers and patients can begin to put together elements of a clinical formulation. For instance, a patient experiencing intermittent leg weakness 
might disclose a history of trauma with current symptoms of Post-Traumatic Stress Disorder (PTSD), so that 'leg buckling' occurs in response to particular triggers.

The patient is encouraged to take responsibility for their own rehabilitation with professional support and guidance. By discussing the treatment programmes on offer the clinician can gauge the patient's willingness to engage and come up with an estimation of the patient's suitability for treatment. At the end of the assessment the patients are invited to suggest 2 or 3 goals they might like to work on when they start treatment. Patients accepted for the in-patient programme are contacted approximately 10-12 weeks prior to their admission date to start the QGSH.

The Queen Square Guided Self-help Program 


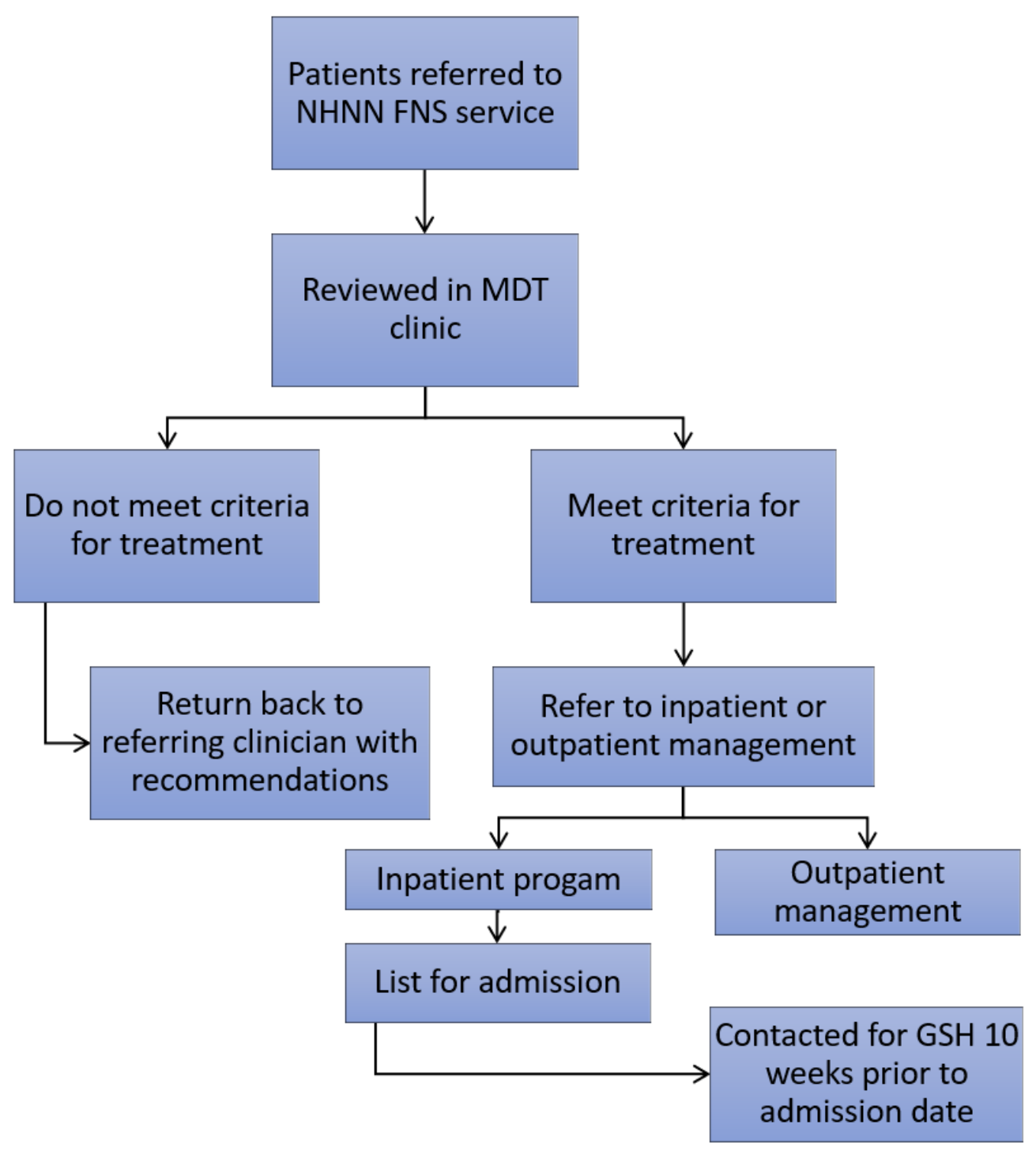

Figure 1: Flow Chart showing the referral pathway for the IP program and GSH

The QGSH is a course of internet-based guided self-help. It runs for up to twelve weeks and includes original videos and patient worksheets, as well as signposting to existing published resources such as Neurosymptoms.org(37). It involves therapists guiding the patient to use range of psychoeducational resources and guides to simple therapy activities, supported by one-to-one contact, such as brief telephone calls, at sparce intervals (35). Resources, including the book 'Overcoming FNS (36), are used according to clinical judgement and patient collaboration within a flexible protocol. Information is sent weekly by e-mail and content is delivered in the form of eleven modules built around video sessions on Youtube (see Table 2). Patients are supplied with complementary, CBT-based worksheets and exercises and supported by telephone appointments by experienced clinicians: a senior occupational therapist (SH), a consultant psychiatrist in psychotherapy (MM) and a psychologist (CS). Guidance telephone appointments take place at 2-3week intervals. The therapists are able to adjust the rate and ordering of the modules to best meet the needs of each individual patient. Adjustments such as sending resources by post are often made, as patient's ability to use electronic devices varies, especially if they are older. 
The GSH therapists must build trust in a timely manner, explaining how the program works and the importance of a collaborative therapeutic alliance. This also implies a therapy contract and outlines

the boundaries of therapy. During the crucial first few weeks, the GSH therapist must develop a rapport, which can be difficult for patients with a history of negative interactions with health care providers. Symptom diaries and worksheets exercises at the start of GSH ensure that the patient's narrative is 'heard', and their symptoms taken seriously. For patients who feel they have not been believed, this is an important component of the therapy. As well as psychoeducation, the therapist is checking patient acceptance of the diagnosis, as even patients who say they agree with the diagnosis of FNS often harbour doubts, e.g. that their symptoms are really due to another condition, such as Lyme Disease or Mast Cell Activation Syndrome. This is part of the process of exploring the patient's beliefs about what has caused and/or is maintaining their symptoms. They may link them to a recent life event such as bereavement or workplace conflict. There may also be cultural issues such as a belief in evil spirits or that their symptoms are simply 'God's will'. The topics covered are shown in Table 1 and include: Introductory sessions on "What are FNS and How do they affect your life?' and further topics including: What is the five areas approach?, Stress, symptoms and the body, Goal Setting, Helpful and Unhelpful thinking, Illness Symptoms and other people, Managing pain, Fatigue management and Dealing with low mood.

Table 2: QGSH Video Titles

1. Introductory Session 1: What are Functional Neurological Symptoms?

2. Introductory Session 2 -- Body, The role of the autonomic system and of stress, Stress and Symptoms

3. Goal Setting

4. Introduction to the 5 Areas Approach (Symptoms, behaviour and affect)

5. 5 Areas Approach focus on Cognitions Thinking and Feelings

6. Anxiety and FNS

7. Fatigue and pain

8. Presentation of workings of the Inpatient Therapies and the MDT

9. Thinking about the self and others:

Mentalization for FNS

10. Mood problems

11. The role of Medications

12. Avoidance in FNS

Setting 'homework' tasks is a key area to explore difficulties in collaborative therapy relationships that require the patient to take an active role. Homework raises important issues such as the patient delaying the return of their homework because of concerns about 'getting it wrong' leading to criticism or embarrassment. This can be addressed during the GSH to pave the way for the more intensive MDT treatment. The phone-calls are also important for bolstering the patient's motivation for change, by engaging them in the clinical formulation and collaborative empiricism i.e. devising and testing hypotheses(38). For patients who are able to work directly with cognitions, during discussion of a Thought Record, the therapist can gently probe and challenge the patient's unhelpful thoughts. The phone-calls also provide an opportunity to check in with the patient about their 
feelings about the therapy and review progress. The patient is then encouraged to set and work towards early goals in their rehabilitation. Finally, attention is paid to the ending of this phase of treatment, which includes realisation that it is not a 'cure all', and the process of handing over care from the preparatory to the inpatient team. The process of handover is one where the patient is encouraged to have a key role: Rather than being passively 'handed over' within the treatment team, the patient is encouraged to inform the inpatient clinicians who first meet them what they have learnt and achieved during the preparatory therapy. This is important both for informing the clinicians but, a lot more important, giving the patient a key responsibility for their selfmanagement.

\section{The GSH Modules}

GSH videos and worksheets are structured into thematic modules. These are provided to patients electronically and supplemented by phone-calls from the GSH therapists. Video-clinics are being developed to flexibly replace phone-calls. As a patient progresses through available modules, the program is personalised for each patient by the therapist in terms of module ordering and rate of delivery. This is aimed at providing the best possible experience, while managing the complex needs of this patient group. Each module comprises a video session accessed on Youtube, and a set of associated worksheets for the patient to complete. These were produced collaboratively by the therapy team to provide an original set of materials. The worksheets were designed to complement the videos and were based in part on the 5-areas Approach book(36) while respecting the copyright permissions given by its authors. We now describe a module that illustrates how a fairly standard CBT approach is finessed to address the needs of this patient group, a philosophy that pervades all preparatory work.

In the 'Anxiety and FNS' module, the basic principles for discussing anxiety are implemented as follows. (i) Collaborative case conceptualisation, whereby patient and therapist look beyond the list of current symptoms to determine the predisposing, precipitating and perpetuating factors; and (ii) collaborative empiricism whereby patient and therapist pool their experience and knowledge in an ongoing process of generating and testing hypotheses.

Some patients with FNS describe feelings and behaviours recognisable as 'anxiety', but they would not describe themselves as 'anxious', while some symptoms that clinicians recognise as anxiety are simply direct bodily experiences far from psychological concepts. 'Anxiety' in the context of FNS is complex and needs to be explored as it can be a triggering factor, or a consequence of symptoms. One patient may recall that everyone in the family was anxious as a result of a tragedy such as the death of a child, while another may recall childhood anxiety alongside other difficulties such as 
elective mutism. Patients who have lived through trauma may be experiencing the symptoms of PTSD, while others may have social anxiety, specific phobias, obsessive compulsive disorder, or panic disorder. Anxiety can also be a direct consequence of FNS. Symptoms such as intermittent leg weakness, numbness or paralysis can cause embarrassment, anxiety, panic, and social isolation. Over time this can drive negative cognitions and low mood.

The 'Anxiety \& FNS' GSH component has two short videos and a worksheet. The videos are a presented as slides and a small 'talking head' in the corner (Fig 2). The sections are: (i) What is anxiety and why is it a normal part of life?, (ii) How anxiety manifests, (iii) When is anxiety helpful and when is it not helpful, (iv) Anxiety in people with FNS, (v) Ways you can help yourself, (vi) An example, and (vii) A little exercise for you to do. The first video aims primarily to educate the patient while the second video is more interactive. The concept of the vicious cycle that causes stress is worked through using an example. The viewer of the video is invited to think of the interplay of cognitions, moods, and bodily symptoms they might experience whilst sitting in the dentist's waiting room. The last section 'A little exercise for you to do', ties in with the accompanying worksheet (Fig 3) and contains a short mindfulness task. The patient is invited to rate their anxiety on a 0-10 scale, then focus on something in the natural world such as a tree or a leaf or flower. They are asked to focus on this object and observe the fine details, then re-rate their anxiety on a 0-10 scale. The final exercise is a symptom diary which asks the patient to develop the habit of analysing their thoughts, emotions, and behaviours at the point of symptom onset.

The 'Anxiety \& FNS' module has been well-received as many patients with FNS have not previously made the link between anxiety, stress, and their symptoms. Sharing the example of the dentist's waiting room can open up a fruitful discussion. During the course of the QGSH, some patients have

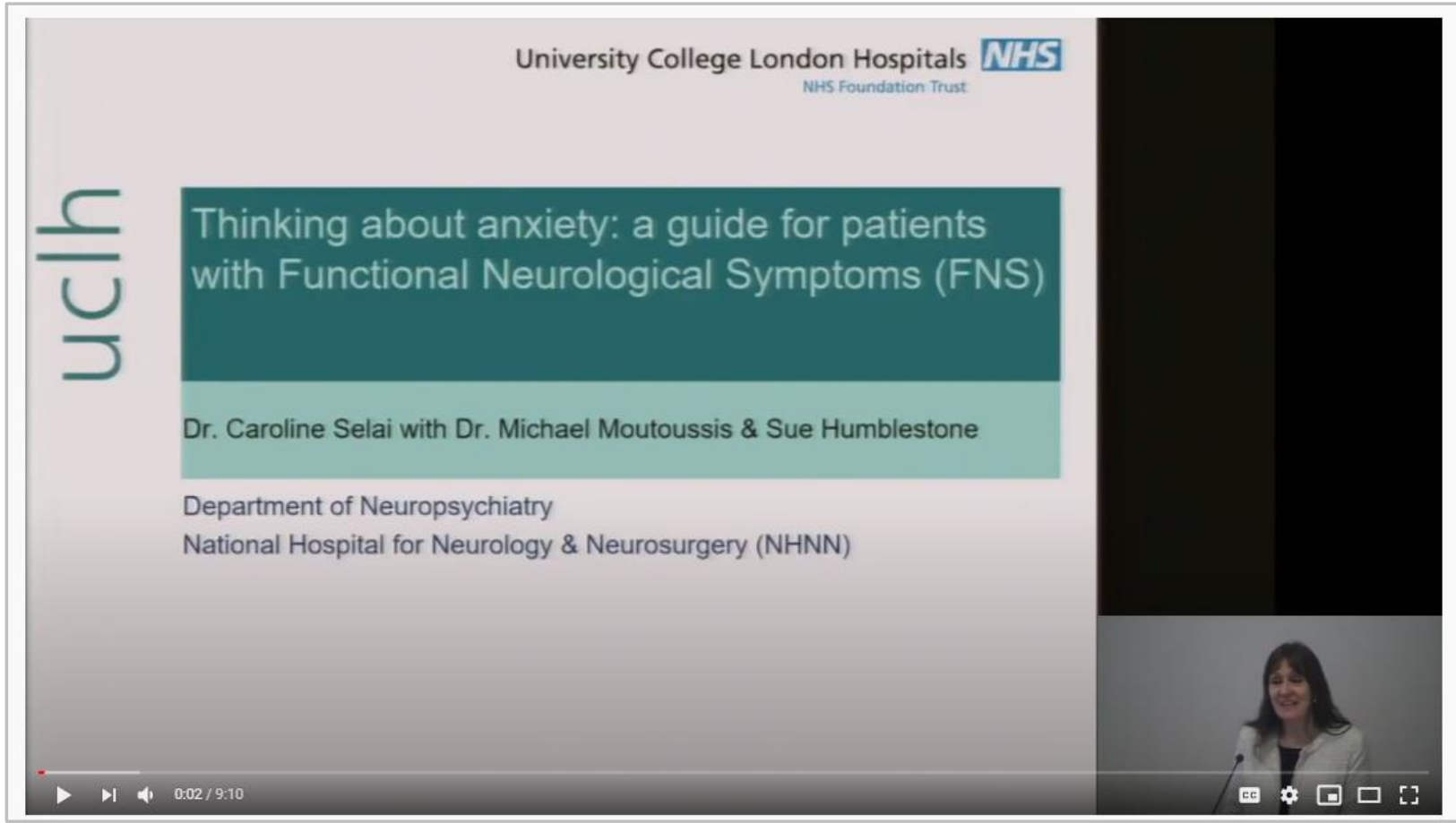

Figure 2: The first video for the 'Anxiety and FNS' (Consent obtained to use photo) 
expressed a wish to 'see' the therapist they are interacting with, and the talking head embedded in the video allows them to see the therapist talking through the slides. The format and delivery of this and other modules is subject to ongoing informal and formal patient evaluative feedback. 
Thinking about anxiety: a guide for patients with Functional Neurological Symptoms (FNS)

Thinking about anxiety: A guide for patients with Functional Neurological Symptoms (FNS) to accompany videos 1 \& 2 .

Dr Caroline Selai \& Dr Michael Moutoussis

This is a worksheet to accompany a section of our Guided Self Help (GSH) on the topic: Thinking about anxiety: a guide for patients with Functional Neurological Symptoms (FNS) There are two videos: Part $1 \&$ Part 2.

As you watch the videos you will see reference made to 3 tasks:

Task 1: Asks you to imagine going to an appointment with your dentist and to write down your thoughts.

Task 2: Invites you to rate how you are feeling on a 0-10 scale.

Task 3: Asks you to compete a diary over the coming week.

These tasks are to help you to engage with the videos and help you to reflect on how life is for you.

- There are no right or wrong answers.

- You might like to get a pen or pencil now, so you have something ready to write with!

- Are you ready to watch the videos...?

- Press Play and let's begin

Thinking about anxiety: a guide for patients with Functional Neurological Symptoms (FNS)

Task 2: When you are feeling stressed or anxious or panicky:

Rate your feelings on a 0-10 scale:

Write down the number..........

Spend a few minutes doing something relaxing such as connecting with something in the natural world. Look at a tree or a flower. What do you notice about the leaves, the petals...?

Breathe deeply. Hold your breath and count: 1, 2, 3 .

Now breathe out.

Rate your feelings on a 0-10 scale:

Write down the number...........
Thinking about anxiety: a guide for patients with Functional Neurological Symptoms (FNS)

Task 1: In the video you are invited to think of a time when you had an appointment with your dentist. Here is the scenario:

You made an appointment with your dentist because of a problem with your tooth. You are in the dentist's waiting room and you will soon be called in. You can smell the mouthwash and you remember that you are always invited to rinse your mouth at the end of treatment.

You start to have automatic thoughts.

- Can you think of some thoughts? Please note them down on your worksheet:

These thoughts lead to bodily sensations.

- Can you think of some of the bodily sensations? Please note them down.

This might affect your mood.

- Can you think of ways your mood might be affected? Please note these down.

Finally, all this might affect your behaviour. What might you do..? Please note this down.

Thinking about anxiety: a guide for patients with Functional Neurological Symptoms (FNS)

Task 3:

Please keep a diary over the next week. Each time you feel 'panicky' please note down your:

- Thoughts

- Emotions

- Physical sensations

- Behaviour

\begin{tabular}{|c|c|c|c|c|}
\hline $\begin{array}{l}\text { Time when I felt } \\
\text { panicky (Date) }\end{array}$ & Thoughts & Emotions & \begin{tabular}{|l|} 
Physical \\
sensations
\end{tabular} & \begin{tabular}{|l} 
Behaviour \\
\end{tabular} \\
\hline & & & & \\
\hline & & & & \\
\hline & & & & \\
\hline & & & & \\
\hline & & & & \\
\hline & & & & \\
\hline & & & & \\
\hline & & & & \\
\hline & & & & \\
\hline & & & & \\
\hline & & & & \\
\hline & & & & \\
\hline & & & & \\
\hline
\end{tabular}




\section{Evaluation and Outcome Data}

In the 35 months the program has run from January 2017 to December 2019, 191 patients have taken part in the inpatient FNS program, and 122 of these had taken part in the QGSH. The rate of completion of the QGSH varied between patients but for this data 'taken part' is defined as at least one email response by the patient. Demographic information is summarised in Table 3.

All patients referred to the IP program were referred to the QGSH, but in small number of patients there were issues with literacy, access or, unusually, urgency of admission leaving no time for QGSH. Patients who did not respond to the invitation email nevertheless progressed to the inpatient program, i.e. QGSH did not have a screening role.

\section{Table 3: Demographic data on patients who took part in the FNS program}

\begin{tabular}{|l|l|}
\hline Patients admitted to inpatient FNS program & 191 \\
\hline Male (\%) & $63(33 \%)$ \\
\hline Female (\%) & $128(67 \%)$ \\
\hline Patient who took part in GSH & 122 \\
\hline
\end{tabular}

When the online service was first used patients reported informally that interacting with the therapists reduced their anxieties about FNS treatment, in particular their concerns about stigma and the attitude of staff. A number of patients talked about their negative experiences of diagnosis and unhelpful interactions in healthcare professionals. Conversations with therapists explored these experiences and helped to reassure these patients. The PROM developed for formal evaluation of the service is composed of 31 items using an ordinal 5-point Likert scale ranging from strongly disagree (1) to strongly agree (5). It was divided into: (A) Knowledge of FNS, (B) Experience using the QGSH materials, (C) Whether QGSH helped the patient transition to the in-patient unit and (D) family involvement in FNS treatment. The CROM is composed of 15 items, again using a 5-point Likert scale, divided into: Knowledge of FNS, Engagement During the QGSH, Handover Organisation, \& Overall Competence for Inpatient Therapy.

The majority of patients have provided positive PROM ratings, particularly in terms of improving their knowledge of FNS and aiding in their transition to the inpatient unit. In the most recent round of data collection (2020) had an overall positive result across all sections, indicating that patients generally perceive the QGSH as useful at preparing them for inpatient therapy. The CROM has been used for four years to assess the perspective of clinicians involved in the inpatient program, and to guide development of the QGSH. Similar to the PROM data most clinicians felt that the QGSH prepared patients well in the area of knowledge of FNS and overall competence for preparatory therapy and has highlighted areas which could be improved in communication and administration. These measures are limited by small data sets but provide initial evidence to support the efficacy and encourage the further development of the QGSH. 


\section{Discussion}

The Queen Square Guided Self-Help program is an internet-based introduction to rehabilitation for Functional Neurological Symptoms, delivered to a group of tertiary care patients with very significant disabilities, by experienced clinicians. Its main aim is to initiate patients to a CBT-based rehabilitation approach, which offers its own clinical benefits but, crucially, mainly aims to optimise the efficacy of subsequent inpatient multidisciplinary treatment. The QGSH has been developed and applied since 2015 and is being evaluated and developed in an ongoing basis. It not only has a clinical impact, but also an academic and educational one, as its associated projects form an excellent arena for graduate student participation in service evaluation.

The experience of the QGSH has highlighted how complex FNS can be to treat, particularly in a tertiary referral centre, and has emphasised the importance of flexible, personalised therapy. To optimise patient engagement and treatment, it has been important for the QGSH therapists to be able to individualize the therapy as to the selection, order and rate of delivery of modules to fit the patient's needs. For the same reason we have found that a taking a cognitive-rehabilitational approach but also developing a psychodynamic understanding of each patient. This consists of a 'psychodynamic prism' of thinking and understanding, rather than directly intervening. It is particularly important for developing a positive therapeutic relationship, and so promoting patient engagement and satisfaction.

The preparatory therapy includes a number of worksheets and videos which are continuously reviewed to ensure that they are clear and easily intelligible. As it is common for this patient group to feel that they have been dismissed or rejected by healthcare professionals in the past, it is particularly important that the videos and worksheets do not use words or phrases which could be interpreted as disrespectful to patients. Part of the review process is therefore to seek feedback that ensures that the language used helps patients feel understood and believed at all points throughout the program. In the long-term, will continue to make the materials easier to use through techniques such as completely electronic worksheets for those patients that prefer them. This should keep in mind ease of access for patients with manual dexterity or (electronic) literacy issues.

Since the inception of the QGSH, we have found it to be an important addition to the inpatient program, providing significant benefit to patients. At the same time, determining the most appropriate outcome measures for this heterogeneous group of patients is work in progress. Many patients have reported that they have found the QGSH very helpful both in and of itself and in helping them to get the most out of the IP programme. The inpatient staff and therapists have also commented that they feel that patients are better prepared the work with the MDT by the QGSH. 
In terms of future prospects, the QGSH provides an excellent springboard for service developments. Prior to the in patient service being paused because of the COVID-19 pandemic in 2020, a total of ten to fifteen patients were taking part in the QGSH at any one time, across the three therapists currently in the team. COVID has demonstrated the need for distanced and virtual approaches to reviewing and treating patients, including in cases where the risks of admission are unacceptable. The QGSH team are in discussions to develop GSH for FNS and medically unexplained symptoms as a stand-alone therapy, to be used as part of a 'stepped-care' approach. Even before the COVID pandemic, the need for a service like this was clear from the increasing waiting list for the inpatient treatment and there is evidence that GSH approaches can be effective in treating anxiety disorders and FNS.

We believe that the set of materials used in QGSH has the potential to provide significant benefit for patients with FNS and can support clinicians wishing to develop their expertise in treating FNS. It could help with the development of new specialist FNS services, for example for less disabled patients where QGSH can form the basis for completely stand-alone interventions where they have not existed before, something we are keen to support. We hope that the experience of the Queen Square team can be used to help patients and clinicians to improve the provision of FNS services. Interested clinicians may contact us at UCLH.NHNN-GSHFNS@nhs.net to discuss access and use of the materials with potential for ongoing collaborations. 


\section{Bibliography}

1. Diagnostic and statistical manual of mental disorders: DSM-5 ${ }^{\mathrm{TM}}$, 5th ed. - PsycNET [Internet].

2. Stone J, Zeman A, Sharpe M. Functional weakness and sensory disturbance. Vol. 73, Journal of Neurology Neurosurgery and Psychiatry. BMJ Publishing Group; 2002. p. 241-5.

3. Ding JM, Kanaan RAA. What should we say to patients with unexplained neurological symptoms? How explanation affects offence. J Psychosom Res. 2016 Dec 1;91:55-60.

4. Carson A, Hallett M, Stone J. Assessment of patients with functional neurologic disorders. In: Handbook of Clinical Neurology. Elsevier B.V.; 2016. p. 169-88.

5. Sojka $P$, Bareš $M$, Kašpárek $T$, Světlák $M$. Processing of emotion in functional neurological disorder. Vol. 9, Frontiers in Psychiatry. Frontiers Media S.A.; 2018.

Stone J, Carson A, Duncan R, Roberts R, Warlow C, Hibberd $C$, et al. Who is referred to neurology clinics? - The diagnoses made in 3781 new patients. Clin Neurol Neurosurg. 2010 Nov;112(9):747-51.

7. Carson AJ, Brown R, David AS, Duncan R, Edwards MJ, Goldstein $\mathrm{LH}$, et al. Functional (conversion) neurological symptoms: Research since the millennium. Vol. 83, Journal of Neurology, Neurosurgery and Psychiatry. BMJ Publishing Group Ltd; 2012. p. 842-50.

8. Gates JR. Non-epileptic Seizures: Classification Coexistence with Epilepsy: Diagnosis, Therapeutic Approaches and Consensus. Epilepsy Behav [Internet].

9. Pick S, Goldstein LH, Perez DL, Nicholson TR. Emotional processing in functional neurological disorder: A review, biopsychosocial model and research agenda. Vol. 90, Journal of Neurology, Neurosurgery and Psychiatry. BMJ Publishing Group; 2019. p. 704-11.

10. Edwards M. Functional neurological symptoms: welcome to the new normal. 2016 [cited 2020 Jan 19];

11. McKenzie P, Oto M, Russell A, Pelosi A, Neurology RD2010 U. Early outcomes and predictors in 260 patients with psychogenic nonepileptic attacks. AAN Enterp [Internet]. 2010 [cited 2020 Jan 19];

12. Karterud HN, Ris $\varnothing r \mathrm{MB}$, Haavet OR. The impact of conveying the diagnosis when using a biopsychosocial approach: A qualitative study among adolescents and young adults with NES (non-epileptic seizures). Seizure. 2015 Jan 1;24(C):107-13.

13. Reuber M, Mitchell AJ, Howlett SJ, Crimlisk HL, Grünewald RA. Functional symptoms in neurology: Questions and answers. Vol. 76, Journal of Neurology, Neurosurgery and Psychiatry. 2005. p. 307-14.

14. Rommelfanger KS, Factor SA, LaRoche S, Rosen P, Young $\mathrm{R}$, Rapaport $\mathrm{MH}$. Disentangling stigma from functional neurological disorders: Conference report and roadmap for the future. Front Neurol. 2017 Mar 29;8(MAR)
15. Sharpe M, Stone J, Hibberd C, Warlow C, Duncan R, Coleman R, et al. Neurology out-patients with symptoms unexplained by disease: Illness beliefs and financial benefits predict 1-year outcome. Psychol Med. 2010 Apr;40(4):689-98.

16. Gelauff J, Stone J, Edwards M, Carson A. The prognosis of functional (psychogenic) motor symptoms: A systematic review. J Neurol Neurosurg Psychiatry. 2014;85(2):220-6.

17. Sharpe M, Walker J, Williams C, Stone J, Neurology JC-, 2011 U, et al. Guided self-help for functional (psychogenic) symptoms: A randomized controlled efficacy trial. Neurology [Internet]. 2011

18. Conwill M, Oakley L, Evans K, Behavior AC-E\&, 2014 $\mathrm{U}$. CBT-based group therapy intervention for nonepileptic attacks and other functional neurological symptoms: a pilot study. Elsevier [Internet].

19. Demartini B, Batla A, Petrochilos P, Fisher L, Edwards $\mathrm{MJ}$, Joyce $\mathrm{E}$. Multidisciplinary treatment for functional neurological symptoms: a prospective study. 2014;

20. Greiner C, Schnider A, Leemann B. Functional neurological disorders: A treatment-focused review. Vol. 167, Swiss Archives of Neurology, Psychiatry and Psychotherapy. EMH Media; 2016. p. 234-40.

21. Schipper $L$ de, Vermeulen $M, \ldots$ AE-C neurology and, 2014 U. Diagnosis and management of functiona neurological symptoms: The Dutch experience. Elsevier [Internet]. 2014

22. Hubschmid M, Aybek S, Maccaferri GE, Chocron O, Gholamrezaee MM, Rossetti AO, et al. Efficacy of brief interdisciplinary psychotherapeutic intervention for motor conversion disorder and nonepileptic attacks. Gen Hosp Psychiatry. 2015 Sep 1;37(5):44855.

23. Saifee TA, Kassavetis $P$, Pareés I, Kojovic $M$, Fisher $L$, Morton L, et al. Inpatient treatment of functional motor symptoms: A long-term follow-up study. J Neurol. 2012 Sep;259(9):1958-63.

24. Jordbru AA, Smedstad LM, Klungsøyr O, Martinsen EW. Psychogen ic gait diso rde $\mathrm{r}$ : A random ized cont rolled trial of physical rehabilitation with one -year fo llow -up. J Rehabil Med. 2014;46(2):181-7.

25. Nielsen G. Physical treatment of functional neurologic disorders. Elsevier [Internet].2016

26. Nielsen G, Stone J, Edwards MJ. Physiotherapy for functional (psychogenic) motor symptoms: A systematic review. Vol. 75, Journal of Psychosomatic Research. 2013. p. 93-102.

27. Nielsen G, Ricciardi L, Demartini B, Hunter R, Joyce E, Edwards MJ. Outcomes of a 5-day physiotherapy programme for functional (psychogenic) motor disorders. J Neurol. 2015 Mar 18;262(3):674-81.

28. Dallocchio C, Tinazzi M, Bombieri F, Arnó N, Erro R. Cognitive behavioural therapy and adjunctive physical activity for functional movement disorders (Conversion Disorder): A pilot, single-blinded, randomized study. Vol. 85, Psychotherapy and 
Psychosomatics. S. Karger AG; 2016. p. 381-3.

29. O'Neal MA, Baslet G. Treatment for patients with a functional neurological disorder (conversion disorder): An integrated approach. Am J Psychiatry [Internet]. 2018 Apr 1

30. Sattel H, Lahmann C, Gundel H, Guthrie E, Kruse J, Noll-Hussong $M$, et al. Brief psychodynamic interpersonal psychotherapy for patients with multisomatoform disorder: Randomised controlled trial. Br J Psychiatry [Internet]. 2012 Jan

31. Nicholson TR, Carson A, Edwards MJ, Goldstein LH, Hallett M, Mildon B, et al. Outcome measures for functional neurological disorder: A review of the theoretical complexities. J Neuropsychiatry Clin Neurosci [Internet]. 2020

32. Marks I. Living With Fear: Marks, Isaac M.: 9780070403963: Amazon.com: Books [Internet].

33. Lovell K, Richards D. MULTIPLE ACCESS POINTS AND LEVELS OF ENTRY (MAPLE): ENSURING CHOICE, ACCESSIBILITY AND EQUITY FOR CBT SERVICES. Behav Cogn Psychother. 2000 Oct;28(4):379-91.

34. Healthcare Improvement Scotland. Stepped care for functional neurological symptoms. Heal Improv Scotl [Internet]. 2012;(February).

35. Cuijpers P, Schuurmans J. Self-help interventions for anxiety disorders: An overview. Vol. 9, Current Psychiatry Reports. Springer; 2007. p. 284-90.

36. Williams C, Kent C, Smith S, Carson A, Sharpe M, Cavanagh J. Overcoming functional neurological symptoms: A five areas approach. Overcoming Funct Neurol symptoms A five areas approach [Internet]. 2011;

37. Welcome - neurosymptoms.org [Internet]. [cited 2020 Jun 18]. Available from:

https://www.neurosymptoms.org/

38. Beck JS, Wright JH. BOOK REVIEWS JOURNAL OF PSYCHOThERAPY PRACFICE AND RESEARCH Cognitive Therapy: Basics and Beyond [Internet]. Vol. 6, The Journal of Psychotherapy Practice and Research. American Psychiatric Publishing; 1997 


\section{DSM V Criteria}

A. One or more symptoms of altered voluntary motor or sensory function

B. Clinical findings provide evidence of incompatibility between symptom and recognised neurological or medical conditions

C. Symptom or deficit is not better explained by another medical or mental disorder

D. Symptom or deficit causes clinically significant distress or impairment in social, occupational or other important areas of functioning or warrants medical evaluation

With specific symptom type:

Motor Weakness or paralysis

Abnormal movement (e.g. tremor, dystonic movement, myoclonus, gait)

Swallowing, Speech

NES Attacks or seizures

Sensory Anaesthesia or sensory loss

Mixed

Special sensory (e.g. visual, olfactory, auditory)

Reproduced from: American Psychiatric Association. (2013). Diagnostic and statistical manual of mental disorders (5th ed.). Arlington, VA: American Psychiatric Publishing, pp. 318 .

\section{ICD-11 for Mortality and Morbidity Statistics (Version : 04 / 2019)

\begin{tabular}{|c|c|}
\hline Search Functional Neurological Symptom Disorder & ? [ Advanced Search] \\
\hline
\end{tabular}

6B60.Z Dissociative neurological symptom disorder, with unspecified symptoms

All ancestors up to top

- 06 Mental, behavioural or neurodevelopmental disorders

- Dissociative disorders

- $6 \mathrm{~B} 60$ Dissociative neurological symptom disorder

- 6B60.Z Dissociative neurological symptom disorder, with unspecified symptoms 